\title{
A search for solar $g$ modes in the GOLF data
}

\author{
A. H. Gabriel ${ }^{1}$, F. Baudin ${ }^{1}$, P. Boumier ${ }^{1}$, R. A. García ${ }^{2}$, S. Turck-Chièze ${ }^{2}$, T. Appourchaux ${ }^{3}$, L. Bertello ${ }^{4}$, \\ G. Berthomieu ${ }^{5}$, J. Charra ${ }^{1}$, D. O. Gough ${ }^{6}$, P. L. Pallé ${ }^{7}$, J. Provost ${ }^{5}$, C. Renaud ${ }^{5}$, J.-M. Robillot ${ }^{8}$, T. Roca Cortés ${ }^{7,9}$, \\ S. Thiery ${ }^{1,10}$, and R. K. Ulrich ${ }^{4}$ \\ ${ }^{1}$ Institut d'Astrophysique Spatiale, Université Paris XI, 91405 Orsay, France \\ 2 DAPNIA/Service d'Astrophysique, CEA/Saclay, 91191 Gif-sur-Yvette Cedex, France \\ 3 Research and Science Support Department of ESA, Keplerlaan 1, 2200AG, Noordwijk, The Netherlands \\ 4 Department of Astronomy, U.C.L.A., Los Angeles, USA \\ 5 Observatoire de la Côte d'Azur, Laboratoire Cassini, 06304 Nice, France \\ ${ }^{6}$ Institute of Astronomy, University of Cambridge, Cambridge CB3 OHA, UK \\ 7 Instituto de Astrofísica de Canarias, 38205 La Laguna, Tenerife, Spain \\ 8 Observatoire de l’Université Bordeaux 1, BP 89, 33270 Floirac, France \\ 9 Departamento de Astrofísica, Universidad de La Laguna, Tenerife, Spain \\ 10 University of Birmingham, Edgbaston, Birmingham B15 2TT, UK
}

Received 12 March 2002 / Accepted 6 May 2002

\begin{abstract}
With over 5 years of GOLF data having some 90\% continuity, a new attempt has been made to search for possible solar $g$ modes. Statistical methods are used, based on the minimum of assumptions regarding the solar physics; namely that mode line-widths are small compared with the inverse of the observing time, and that modes are sought in the frequency interval 150 to $400 \mu \mathrm{Hz}$. A number of simulations are carried out in order to understand the expected behaviour of a system consisting principally of a solar noise continuum overlaid with some weak sharp resonances. The method adopted is based on the FFT analysis of a time series with zero-padding by a factor of 5. One prominent resonance at $284.666 \mu \mathrm{Hz}$ coincides with a previous tentative assignment as one member of an $n=1, l=1, p$-mode multiplet. Components of two multiplets, previously tentatively identified as possible $g$-mode candidates from the GOLF data in 1998, continue to be found, although their statistical significance is shown to be insufficient, within the present assumption regarding the nature of the signal. An upper limit to the amplitude of any $g$ mode present is calculated using two different statistical approaches, according to either the assumed absence (H0 hypothesis) or the assumed presence (H1 hypothesis) of a signal. The former yields a slightly lower limit of around $6 \mathrm{~mm} / \mathrm{s}$.
\end{abstract}

Key words. Sun, helioseismology

\section{Introduction}

\subsection{The internal structure through helioseismology}

Since global oscillations from the Sun were first identified, the challenge has been to exploit their measured frequencies in order to determine the internal structure. Much progress has now been accomplished. Using the observed $p$-mode spectrum, measurements of the outer layers can be determined in considerable detail, yielding the sound velocity and the rotation rate as functions of the radius and the latitude. These quantities are then compared with predictions coming from theoretical numerical modelling of the solar interior, in order to assess the validity of the different physical parameters implicit in the models.

Ground-based helioseismic networks, such as IRIS (Grec et al. 1991), BiSON (Chaplin et al. 1996) and GONG (Harvey et al. 1996), have accumulated 10 or more years of global

Send offprint requests to: A. H. Gabriel, e-mail: gabriel@ias.fr oscillations data, albeit with inevitable interruptions. Since the launch of SOHO, these have been augmented by over 5 years of space-based observations coming from the instruments MDI (Scherrer et al. 1995), VIRGO (Fröhlich et al. 1997) and GOLF (Gabriel et al. 1997), with a substantial improvement in continuity and the elimination of terrestrial atmospheric perturbations. Each of the instrument teams has now published inversions of their $p$-mode frequencies in terms of the radial variation of sound speed in the interior.

The determination of core structure from $p$-mode frequencies is a basically ill-conditioned inversion problem. Only the low-degree modes reach the core, of which only the $l=0$ mode reaches the solar centre. However, all of the $p$ modes, including the $l=0$ modes, have their major contribution in the outer layers of the Sun. Inversion of $p$-mode frequencies to produce core structure requires the inclusion of mode degrees up to $l$ of around 300, in order to compensate correctly for the large outer contribution to the $l=0$ and 1 modes. It follows from this 
ill-conditioning that the precision required for the input data (frequencies) is very high. To determine core sound speed to $10^{-3}$ precision requires a frequency precision for 5-minute $p$ modes of the order of $10 \mathrm{nHz}$ or better. This high precision is equally important for the higher- $l$ modes involved in the inversion, although they are not present in the core.

Instruments using data integrated over the solar disc, such as GOLF and SPM/VIRGO cannot measure reliably $l$ values above 3 or 4 because of the reduced sensitivity of non-imaged observations to these modes and must depend therefore for the higher $l$ on complementary data from spatially resolved observations, such as LOWL (Tomczyk et al. 1996), GONG or MDI. A further problem concerns the difficulty of measuring the precise resonant frequencies, due to their non-Lorentzian shape on a frequency scale. Several workers (e.g. Duvall et al. 1993; M. Gabriel 1993; Abrams \& Kumar 1996) have shown that the resonances will have an asymmetric form. More recently, efforts have been made to fit the observed data, taking the asymmetry into account (Toutain et al. 1998). For GOLF, Thiery et al. (2000) and Bertello et al. (2000a) have redetermined $p$-mode frequencies with a higher accuracy by fitting asymmetric profiles to the resonances. Regrettably, asymmetric profile fitting is much more difficult for the higher- $l$ spatially resolved data and is not yet generally available. We should therefore assume that this absence is limiting the accuracy of the current best available inversions, of which the most recent is based upon GOLF asymmetric low- $l$ and MDI symmetric higher- $l$ frequencies (Basu et al. 2000).

\subsection{Importance of $g$ modes}

In spite of the considerably improved frequency precision obtainable today for $p$ modes from GOLF and others, the above limitations are leading to only a modest advance towards improving the precision for the core. This may also be due to a number of other factors: inevitable variation of the $p$-mode frequencies with the solar cycle, uncertainties in the influence of solar surface effects on the frequencies and limitations implicit in the presently used techniques for inversion. Progress on some of these points will be made when we are able to exploit the frequencies of lower-order $p$ modes with $n$ less than 10 . Some of these are now beginning to be observed and included in the analyses. (see Bertello 2000b; García et al. 2001a; TurckChièze et al. 2001). However, the situation would be greatly improved if we were able to use the $g$-mode frequencies.

In contrast to the $p$ modes, the gravity or $g$ modes are confined largely to the radiative zone, with their maximum of energy near the centre of the Sun. For this reason, the inversion of $g$-mode frequencies for the core structure would be a well conditioned procedure. Higher-precision results could in principle be obtained from lower-precision input data, and the demands placed on the rigour of the inversion procedure are much less. The difficult surface problem is also avoided. For similar reasons, the $g$ modes would also give more direct and precise answers for the core rotation rate. In spite of several reported claims in the literature (e.g. Delache \& Scherrer 1983; van der Raay 1988; Thomson et al. 1995), no observation of solar

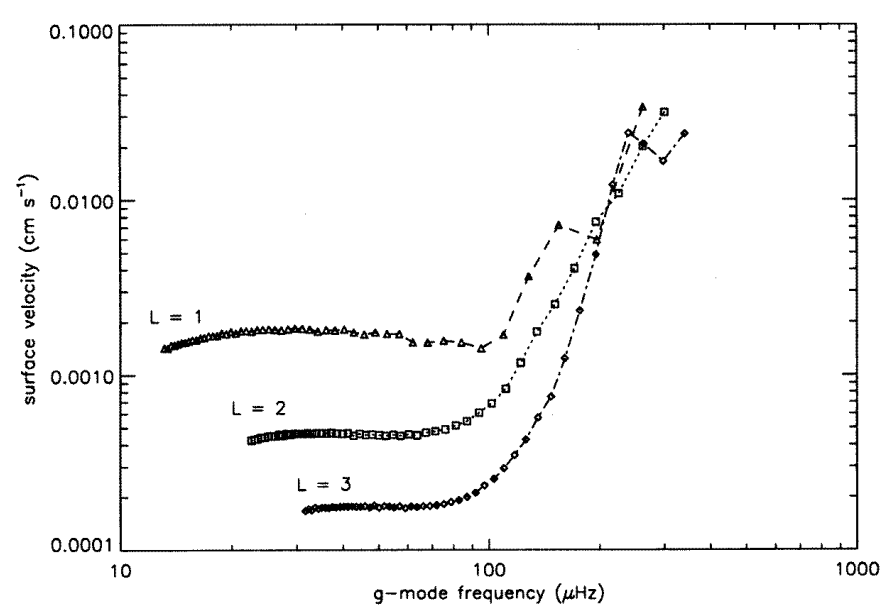

Fig. 1. Predicted surface velocity amplitudes for solar $g$ modes, according to Kumar et al. (1996).

$g$-mode oscillations has been reliably confirmed. Gravity modes are predicted with frequencies up to about $400 \mu \mathrm{Hz}$. A theoretical estimate by Kumar et al. (1996) shown in Fig. 1 predicts $g$-mode amplitudes of less than $0.4 \mathrm{~mm} / \mathrm{s}$. Earlier similar predictions were reported by Gough (1985), and assume that the pertinent $g$ modes are excited principally via their interaction with the turbulent motion in the upper superadiabatic boundary layer of the convection zone. Such a low amplitude would be out of reach of present day helioseismic observations. It should be realised that there may be other excitation mechanisms that are not well understood (see, for example, Gavryusev \& Gavryuseva 1988) which would augment the amplitudes. In view of the large uncertainty implicit in all of these estimates, the search for $g$ modes remains a serious activity at the present time. It is noteworthy that, as can be seen from Fig. 1, the highest power levels are predicted for the higherfrequency, low-order modes.

The GOLF team has reported earlier a search which identified two possible $g$-mode candidates (Gabriel et al. 1998), with velocity amplitudes around $7 \mathrm{~mm} / \mathrm{s}$, at frequencies close to some predictions from solar models. At the same time the Phoebus consortium (representing VIRGO, MDI, and BiSON) announced a null observation, with an upper limit of $1 \mathrm{~mm} / \mathrm{s}$ (Fröhlich et al. 1998). The Phoebus consortium have now revised their analysis and modified their upper limit value to $10 \mathrm{~mm} / \mathrm{s}$ (Appourchaux et al. 2000). This later result is not in conflict with the present analysis from GOLF.

\subsection{The identification problem}

As will be shown later, the expected frequency region for the $g$ modes is dominated by a velocity background spectrum arising from a combination of the random fields of solar convection cells of all scales. This produces a continuum in the frequency spectrum with a characteristic shape and statistics.

Today, most observers would agree on the basic parameters of the problem. We are trying to identify possible $g$ modes, having an amplitude which cannot be more than 7 to $10 \mathrm{~mm} / \mathrm{s}$, against an intrinsic background noise level which is, at these 
frequencies, observed to be around $1 \mathrm{~mm} / \mathrm{s}$ per frequency resolution element, and believed to be predominently due to solar noise. In this situation, it is difficult to identify $g$ modes unequivocally unless some expectation parameters based on solar theory are taken into account. In the present paper we aim to limit such assumptions to the strict minimum, in order to maintain the maximum of objectivity in searching for resonant frequencies that can be distinguished from the background noise and to avoid, as far as possible, identifications biased by unverified solar theory. Alternative approaches are also being examined. A complementary study is in hand (Turck-Chièze et al., submitted to ApJ), which aims to exploit the statistical advantages of searching for multiplet structures. This approach has been described by Turck-Chièze et al. (1998).

In the next section, we describe the GOLF data used in the present study. The subsequent section treats the nature of the signal and background. This is followed by a discussion of some of the methods available for identifying candidate $g$ modes, supported by appropriate simulations. The application of the selected approach is then presented, followed by a discussion of the results obtained.

\section{Selection of the GOLF data set}

At this point, we have available a set of GOLF data covering the period 1996 April 11 to 2001 June 11, a total of 1888 days, with some gaps totalling 176 days, notably the one associated with the temporary loss of the SOHO spacecraft in the summer of 1998. Data are available from only one wing of the sodium line profile, following a decision in April 1996 to switch off the unreliable polarizing modulation mechanisms. The blue wing was selected at this time. However, at the second switch-on, following the recovery of SOHO in September 1998, it proved to be safer and faster to set GOLF on the red wing, where it has since remained.

In combining time series from the blue and red wings, a question arises concerning their relative phases. Several studies have been made of this issue, using the 6-week period of GOLF 2 -wing data obtained during the commissioning period. For the 5-minute $p$ modes, Pallé et al. (1999), Régulo et al. (1998) and Renaud et al. (1999) have examined the phase relation, and have shown that the phase difference is not the $180^{\circ}$ that would be expected for a simple Doppler translation of the profile on a wavelength scale, but closer to $163^{\circ}$. This is also confirmed recently by Thiery et al. (2001) in comparisons of phase between GOLF and MDI. The remaining $17^{\circ}$ is expected to have two sources. The first is a straightforward contamination of the velocity measurement by oscillations in intensity. When analysis is carried out on one wing only, the signal is sensitive to oscillations in the level of the continuum from which the absorption is measured. This effect is expected to add a contribution having a $0^{\circ}$ phase between the two wings (instead of the $180^{\circ}$ for the velocity component), but $\sim 90^{\circ}$ displaced from the velocity signal. However, using the intensity oscillations observed by, say, VIRGO, we can estimate the importance of this effect to be such that the resultant red-blue phase difference would be expected to fall only by around $2^{\circ}$. The second source of phase shift lies in the error of assuming that the profile is simply dis- placed in wavelength due to the Doppler effect. In reality, it also changes in shape, as reported by Robillot, who observes the effect in his 5-point instrument MR5 (Robillot et al. 1993) and who termed the effect "palpitation".

Our basic calibration process corrects automatically for the $180^{\circ}$ arising from the opposite slopes on the two wings of the profile, so that we discuss here only the residual $17^{\circ}$. We can either correct for this $17^{\circ}$ before combining the two sections of the data, or we can ignore it. Ignoring the $17^{\circ}$ phase shift does not in reality change the amplitude of candidate modes significantly (see García et al. 2001b, where a $36^{\circ}$ shift produces only a modest effect). This simple solution is the one we have chosen to adopt here.

It should also be remembered that the contribution from different points on the solar disc to our integrated solar velocities can be quite complex. Calculated contribution functions (García 1996; Henney et al. 1999) show significant E-W asymmetry when only one wing is used. The $17^{\circ}$ shift observed for some of the 5-minute $p$ modes may not be valid for lower frequencies, nor for other $l$-values, nor for $g$ modes, which have a different relation between vertical and horizontal motions. Nevertheless, the E-W asymmetry is not large, and it seems unlikely that much larger red-blue phase differences will be found for other modes. Following these arguments, a simple combination of the blue and red time series with zero phase adjustment should be an acceptable solution.

The GOLF spectrometer has two photomultiplier detectors, designated PM1 and PM2. These should receive identical signals, and their outputs are often added together in order to increase the photon signal-to-noise ratio. However, there are some indications from another diagnostic channel that PM1 is suffering from some very small gain fluctuations of unknown origin. We choose in the present work to use only the data from PM2, thereby accepting a small increase in the photon noise level, with the aim of improving data reliability.

The conversion of GOLF data from Level 1 (intensities measured in the wing of the solar absorption line) to Level 2 (a time series of line-of-sight global velocities) is a non-trivial problem, made more difficult by the absence of simultaneous measurements on both wings of the line profile. We currently use Level 2 data derived using three separate sets of data reduction, developed within the GOLF consortium. These arise from the use of calibration techniques described by Ulrich et al. (2000) (set A), García et al. (article in preparation) (set B) and Grec (private communication) (set $\mathrm{C}$ ). We find that, for the purposes of the present analysis, these data sets are closely similar. Detailed comparisons show that, in the frequency band presently considered, all of the stronger peaks appear in each of the spectra, with a precise frequency agreement. However, the precise power level of these peaks varies slightly between the sets. The variation is random for each bin in the spectrum, and increases with decreasing frequency. In the vicinity of $300 \mu \mathrm{Hz}$ it has a standard deviation of $12 \%$ in spectral power units for the larger peaks. We draw the conclusion that this figure represents an uncertainty in the measured power, arising from the limitation in our calibration techniques. Although we have carried through the analysis using different data sets, the 
work presented in the remainder of this paper is based on the analysis using the calibrated data of set $\mathrm{A}$.

\section{Signal and background}

The basic problem is one of searching for $g$-mode global solar oscillations in the presence of a background or "noise". In order to distinguish between the two contributions to the measured signal, we need to define certain expected properties on the nature of each. Starting with the $g$ modes, since these have never previously been observed, we choose here to impose the minimum of constraints on their likely properties. This minimum assumption is that their life-times are long compared with the duration of a few years of our observations (see Hill et al. 1991 and references therein). The only other constraint is to limit the search to the frequency range $150 \mu \mathrm{Hz}$ to $400 \mu \mathrm{Hz}$, where we expect to find the strongest $g$ modes, as suggested by Turck-Chièze et al. (1998). The long life-time assumption is here assumed to imply that, in an FFT spectrum, almost all of the energy from a single $g$ mode will fall in either one bin or, at most, in two neighbouring bins, depending on the relation between their precise frequencies and those of the discrete bins (see also Sect. 5.3). It also follows from this assumption that we cannot obtain from the observations information on their actual life-times or damping rates, so that it is pointless to attempt to fit profiles to the suspected resonances.

The background noise will in general be composed of three components. In decreasing order of expected importance, these are:

1. Random solar noise, due to convection and other noncorrelated local motions of the solar photosphere. This is the solar background, as predicted by Harvey (1985). The statistical characteristics of this component are discussed in Sect. 4.

2. The instrumental photon noise, due to the finite counting rate of the detector. This should be a flat spectrum and becomes important compared with the solar noise only at high frequencies. It should have strictly random statistics $\left(\chi^{2}\right.$ on a power scale). In the case of GOLF, it is estimated to be a factor $\sim 20$ below the solar noise in the $g$-mode region.

3. Instrumental noise of a specific origin. This might be in the form of a continuous spectrum, arising from the Fourier transform of a number of random changes of instrument sensitivity. Alternatively, and more dangerous, are specific instrumental frequencies due to periodic effects in the instrument or in its spacecraft environment. Such frequencies are frequently observed in space instruments and, unless identified as such, might be indistinguishable from solar global oscillations.

Since the major background component is the intrinsic solar noise, it is sometimes assumed that the possibility of detection of $g$ modes will be largely independent of the particular instrument considered. We would argue that, because of the possible existence of specific instrumental noise, both continuous and discrete, which is difficult to detect, this may not be the case, in spite of the superficially similar appearance of the dominant solar noise spectrum shape in the data from different instruments.
Great care has been exercised at all stages in the development of GOLF to ensure the minimum of perturbation and the maximum of data continuity (Gabriel et al. 1995). Data calibration methods have aimed to ensure the correction of effects due to small changes in temperature or other instrument parameters. Uniquely for GOLF, no evidence of any spurious peaks has been found at any of the known SOHO spacecraft parasitic frequencies. For these reasons, we hope for significant advantages from the GOLF instrument.

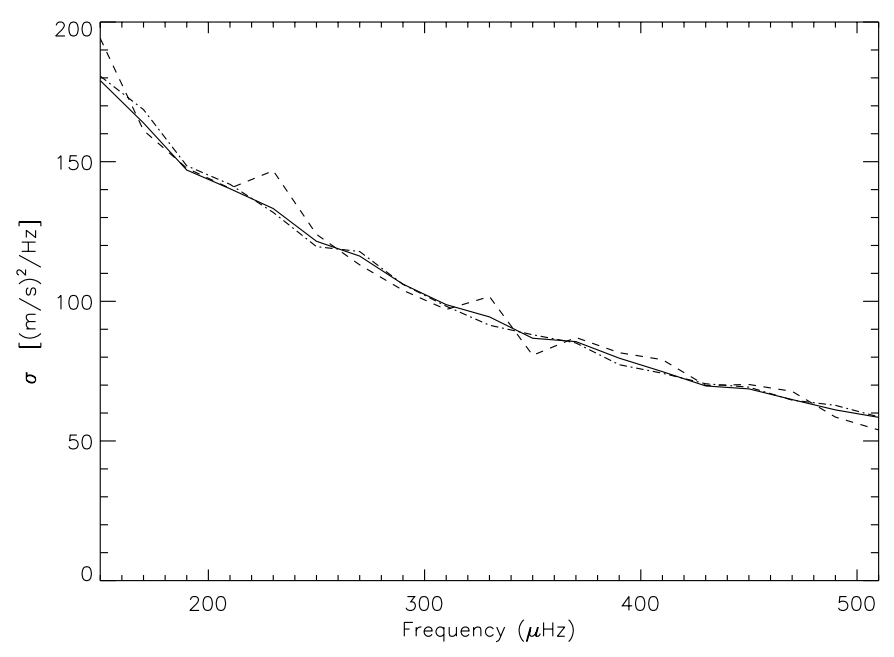

Fig. 2. Showing the agreement between three methods of determining the mean background level, as a function of the separate $20 \mu \mathrm{Hz}$ intervals. This indicates also the absolute level of the background, believed to mainly of solar origin.

\section{Characterising the noise background}

As indicated in the previous section, the background is made up of the three itemised contributions, with the solar component being dominant. It is usual for workers in this domain to assume that this solar contribution is statistically random in nature, and can therefore be treated using Gaussian statistics, equivalent to $\chi^{2}$ statistics for a power spectrum representation. This assumption is critical in estimating whether the quantitative probability that the occasional large spikes observed are within statistical limits or are significant exceptions. However, the physical nature of these motions, based on non-linear turbulent convection, does not lead inevitably to a Gaussian distribution. In the present work, we aim to carry out a limited verification of the validity of the Gaussian assumption.

The superposition of random statistical noise from the Sun with the inevitably statistical photon noise from the detector leaves the resultant also with a Gaussian statistical distribution. In reality, we have access to only one solar spectrum, covering the total period of observation, a fact which formally prohibits the study of its probability statistics. The usual approach is to study the distribution of different bins in this frequency spectrum, as indicators of the probability distribution for each individual bin. This is valid only if each bin has the same average level, which is clearly not the case for the Sun, where the mean level increases with decreasing frequency, as predicted 
by Harvey (1985). We choose here not to adopt the often-used technique of "whitening" the background spectrum. We prefer to carry out our analysis over limited regions of the spectrum of some $20 \mu \mathrm{Hz}$ each, in each of which we can reasonably approximate to a constant value for the mean level. This approach avoids the need to justify an assumed shape for the smoothed background spectrum.

We need first to show how one can derive the probability of detecting a pure sine wave buried in a noise having $\chi^{2}$ statistics. The presentation depends on the method adopted for normalization of the Fourier spectrum, which can vary between users. This question is often ignored as it has no direct effect on the result, other than on the scaling of the power spectrum. With certain forms of normalization, the power of a sine wave will be constant independent of the length of the time series, whereas the power of the background (Gaussian) noise will decrease as the series increases in length. We choose here to use the Scargle (1982) definition, which yields a constant power for the noise and an increasing power for a sine wave when the length of the series is increased:

$P(\omega)=\frac{\delta t}{N}\left[\left(\Sigma_{j=1}^{N} X_{j} \cos \omega t_{j}\right)^{2}+\left(\Sigma_{j=1}^{N} X_{j} \sin \omega t_{j}\right)^{2}\right]$

where $N$ is the number of points of the time series $X_{j}$ and $\delta t$ the sampling time. Using this normalisation for a single time series made of a signal $S\left(t_{j}\right)=A_{0} \sin 2 \pi v_{0} t_{j}$ and a Gaussian noise $\mathcal{N}$ of variance $\sigma_{0}^{2}$, we obtain in the power spectrum for a series of $N$ points:

$P_{S}=N \delta t\left(\frac{A_{0}}{2}\right)^{2}$ and $P_{\mathcal{N}}=\sigma_{0}^{2} \delta t$.

In the case of a power spectrum, the distribution of the power of pure noise follows a $\chi^{2}$ distribution with two degrees of freedom, which is a simple exponential distribution:

$\operatorname{PDF}(z) \mathrm{d} z=\frac{1}{\sigma_{0}^{2} \delta t} \exp \left(-\frac{z}{\sigma_{0}^{2} \delta t}\right) \mathrm{d} z$.

Thus, the distribution depends directly on $\sigma_{0}^{2}$, the variance of the noise. This parameter will influence all calculations on the statistical behaviour of the noise, and has therefore to be confirmed reliably. It can be derived from:

1. the mean level of the continuum $\left\langle z>=\sigma_{0}^{2} \delta t=\sigma\right.$,

2. the mean level of random fluctuations of the continuum $<z^{2}>-<z>^{2}=\left(\sigma_{0}^{2} \delta t\right)^{2}$

3. a fitting of the expected exponential curve to a histogram of the pulse height distribution.

The mean value we call here $\sigma$ in common with previous workers. This nomenclature is not strictly correct, since $\sigma$ is a mean value of the noise power and is directly related to a variance $\sigma_{0}^{2}$ of the noise amplitude.

The quality of the fitting in method 3 , together with the agreement between the three methods, are then indications of the validity of the Gaussian assumption. This has been carried out for 18 intervals of $20 \mu \mathrm{Hz}$ which cover the expected $g$-mode range. The results shown in Fig. 2 demonstrate that there is no significant systematic variation between these three estimates.
The quality of the exponential fit to a histogram can best be demonstrated by plotting it on a log/linear graph, where the fit is expected to be a straight line. This is shown in Fig. 3 for selected bands, together with the typical scatter range expected for 3 standard deviations for each point of the histogram. The appropriate statistics in this case for the expected deviations from a straight line are those of a Poisson distribution.

This $\log /$ linear plot offers a useful way of visualizing the conformity, together with what would be the effect of the presence of some individual $g$-mode spikes on the evaluation of the background $\sigma$ values. If the Kumar theory for the $g$-mode power (see Fig. 1) is qualitatively valid, then we might expect to find a small number of the higher-frequency $g$ modes appearing in some of the $20 \mu \mathrm{Hz}$ histograms. These might be evident, either outside or inside the 3 standard deviation level. In either case, they will not affect the fitting of the straight line (exponential), since we have given this fitting a weighting equal to the number of points per histogram bin, so that it is largely dominated by the distribution of the large number of small pulses. In the case of an important number of large $g$-mode spikes (regrettably not our situation!), these $\log$ /linear histogram plots would show clearly that a straight line fitted through the smaller values fails to reproduce sufficient event numbers for the larger pulses.

It might also be argued that the large number (approaching infinity) of weaker $g$ modes expected in the asymptotic low frequency limit could also contribute to a false background $\sigma$ reading. As the present analysis goes down only to $150 \mu \mathrm{Hz}$, we shall not be troubled by this asymptotic region.

Having determined a good local value for $\sigma$, Eq. (3) enables us to derive the probability that the power in one bin is greater than $m$ times the mean value $\sigma$ :

$P(m)=\mathrm{e}^{-m}$.

For a frequency band containing $N$ bins, the probability that at least one bin has a power greater than $m$ becomes:

$P_{N}(m)=1-\left(1-\mathrm{e}^{-m}\right)^{N}$,

which for $\mathrm{e}^{-m} \ll 1$ approximates to

$P_{N}(m)=N \mathrm{e}^{-m}$

It is important to note that the determination of a power level above which an observed peak has a $10 \%$ or a $1 \%$ probability to be due to noise is dependent on the value of $N$ (i.e. the frequency band over which the probability is expressed). This derivation due to Scargle (1982) is fully consistent with that presented by Appourchaux (1998) and now used by many workers in helioseismology.

\section{Considerations in searching for solar modes}

The simplest formulation of the problem is to consider an FFT of a long uninterrupted time series and to search for evidence that some peaks are observed which are not consistent with the expectation from the background spectrum alone. This is the basic approach that we will follow. 

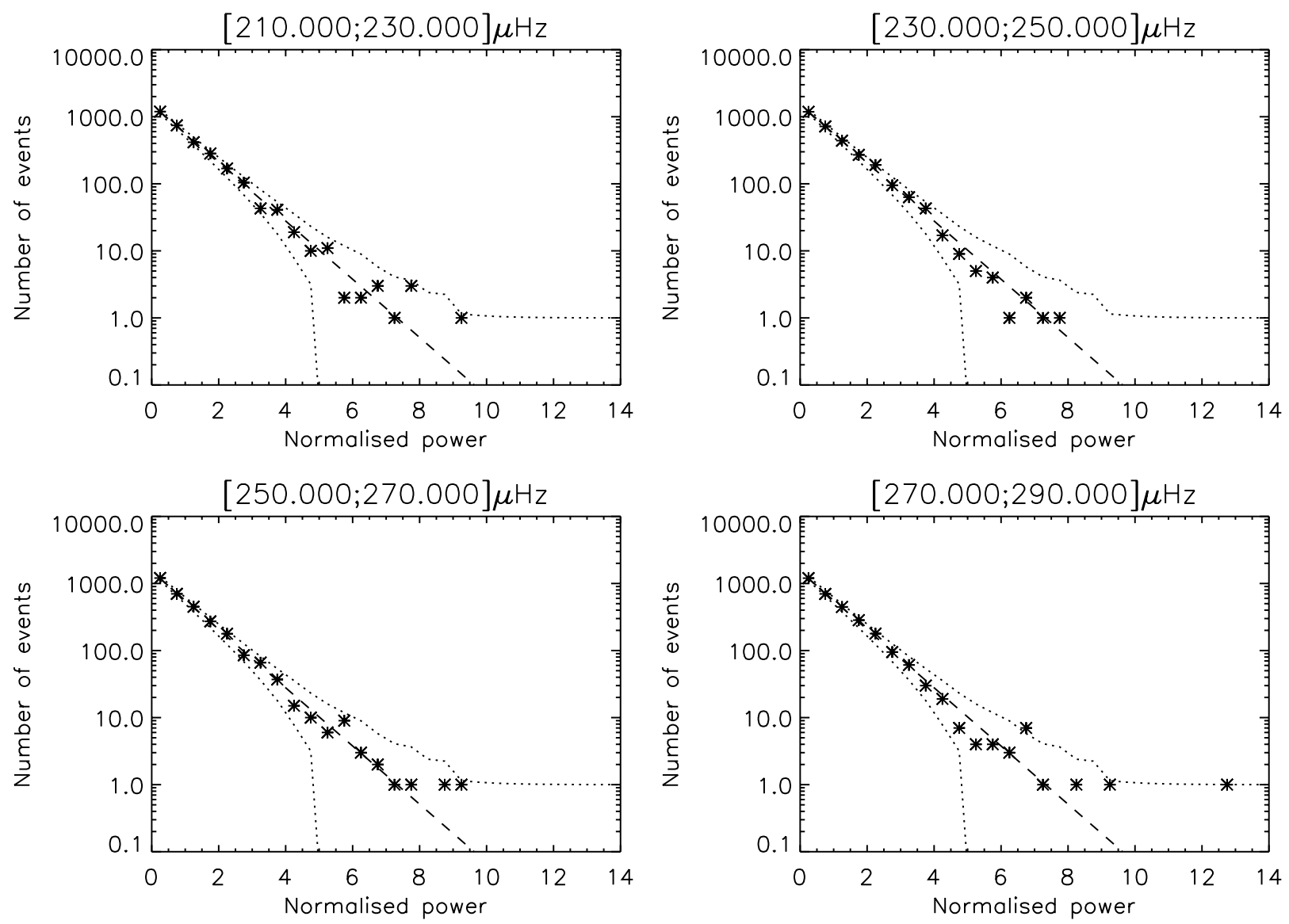

Fig. 3. Showing histograms of the noise distribution in some of the frequency intervals, on a log/linear plot.

We start by using a number of simulations, together with some theoretical concepts, to verify our understanding of the problem. An FFT of a flat spectrum of Gaussian noise background produces a series of discrete bins, each subject to the same probability distribution of power, and each fully independent of its neighbours. A single realization, which is our real situation, gives only one value in each bin. We can deduce the distribution function only by comparing a large number of different bins in a flat part of the spectrum, as has been done above. In the case of simulated data, we are free to produce a large number of independent realizations of the entire spectrum.

For the GOLF time series with $91 \%$ continuity, the gaps are filled with zeros. The bins in the FFT are then no longer fully independent, each Fourier component having a correlation with its immediate neighbours of the order of $9 \%$. This can also produce a small difference between the estimates for $\sigma$ derived from methods 1 and 2, above. We should remember these points, although this does not detract significantly from the basically $\chi^{2}$ distribution for each bin.

\subsection{Nature of the problem}

With the Gaussian background statistics now verified, and the $\sigma$ determined, we turn to the question of how to recognize possible $g$ modes. This consists of determining the largest peaks in the FFT spectrum, and evaluating the probability they might be due merely to statistical noise. To understand better the behaviour of this system, as defined by the foregoing assumptions, we start with some simulations. In Fig. 4 we simulate the effect of combining a random Gaussian solar noise spectrum of $1.7 \mathrm{~mm} / \mathrm{s}$ (our measured value near $300 \mu \mathrm{Hz}$ ) with a coherent sine-wave $g$-mode resonance of $3.5 \mathrm{~mm} / \mathrm{s}$. We choose this amplitude as typical of a signal which might be at the origin of our largest observed peaks, if they were not due to noise alone. Figure 4a shows the two components separately, plotted on a complex (real and imaginary) velocity amplitude plane. The resonance is shown with a specific but arbitrarily chosen phase. The noise is plotted as a probability distribution of dot density, centred around $(0,0)$, achieved by carrying out a large number of realizations. To make the figure more physical, a different normalisation has been used: the vector representing the signal has a length of $A$ and the standard deviation of the noise is $2 \sigma_{0} / \sqrt{N}$.

Figure $4 \mathrm{~b}$ shows the effect of adding these two components, also as a probability dot density. The observed power density of the appropriate FFT bin is just be the square of the magnitude of the vector joining a point in Fig. $4 \mathrm{~b}$ to the origin. Our problem is how to recognize the presence of a resonance, whilst at frequencies either side of the resonance we have only the distribution of Fig. 4a. With only one observational data point at the candidate frequency, we have only one point on the probability plot Fig. 4b. For the background spectrum, however, we have access to the complete distribution of Fig. 4 a (excluding 

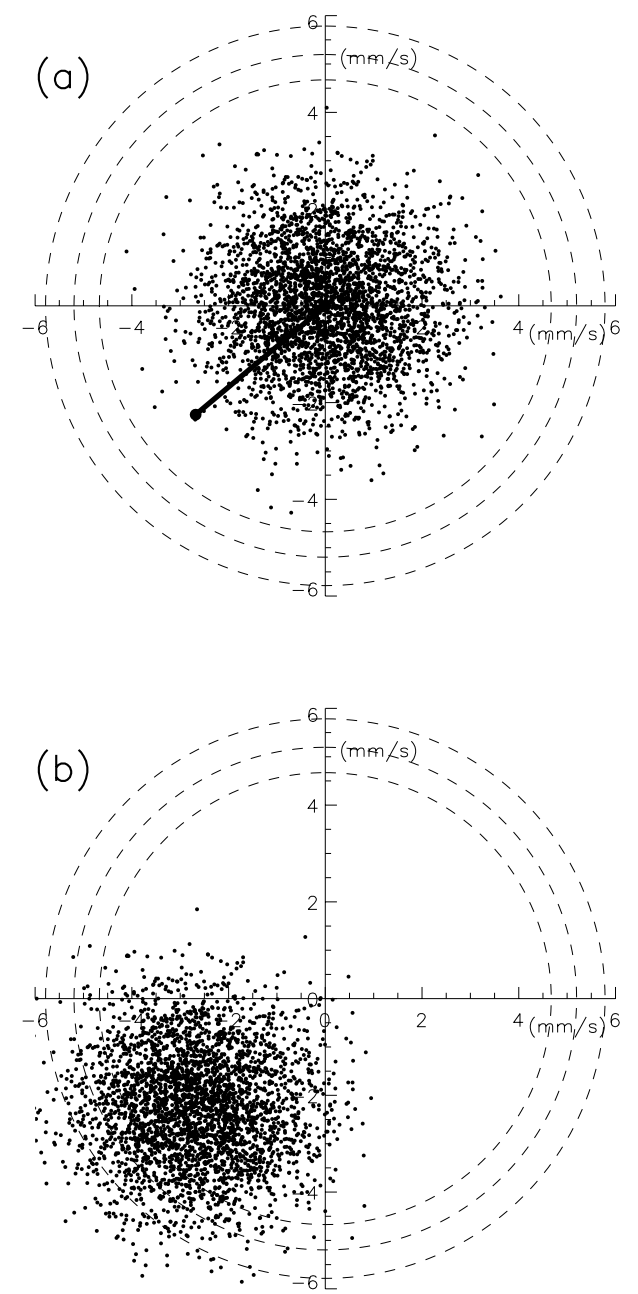

Fig. 4. Simulation representing in the Fourier amplitude plane the effect of a random noise with a standard deviation $2 \sigma_{0} / \sqrt{N} \approx 1.7 \mathrm{~mm} / \mathrm{s}$ and a coherent signal of $3.5 \mathrm{~mm} / \mathrm{s}$. The three dashed circles correspond to the 3 confidence levels of $50 \%, 10 \%$ and $1 \%$. This figure is also a simple illustration of the $\mathrm{H} 0$ hypothesis a) and the $\mathrm{H} 1$ hypothesis b).

the resonance), by observing many different bins. With the parameters here assumed and with a value of $N$ derived from our real data set of 1888 days and a $20 \mu \mathrm{Hz}$ test band near $300 \mu \mathrm{Hz}$ a point in this probability cloud would be regarded as having only a $10 \%$ chance of being due to noise, if it lies more than $5.2 \mathrm{~mm} / \mathrm{s}$ from the origin. This limit is indicated by the second of the three dashed circles superposed in Fig. $4 \mathrm{~b}$.

Thus far, we have considered the probability that a peak, large enough to be prominent, can be due to noise. This is the so-called H0 hypothesis, where the mode amplitude $A$ is taken as zero. The H0 test helps to decide whether to accept or reject its associated hypothesis. The $10 \%$-probability limit used by the Phoebus group (Appourchaux et al. 2000) is based upon the $\mathrm{H} 0$ hypothesis. Here we use also an alternative $\mathrm{H} 1$ hypothesis: that the mode is present $(A \neq 0)$. In this case, the probability is a function of the assumed value of $A$. It can be written as:

$1-P\left(z<z_{0}, \sqrt{N} A / \sigma_{0}\right)$,

where the function $P$ is expressed in terms of the level $z_{0}$ in the power spectrum. This is developed in Appendix A. The two

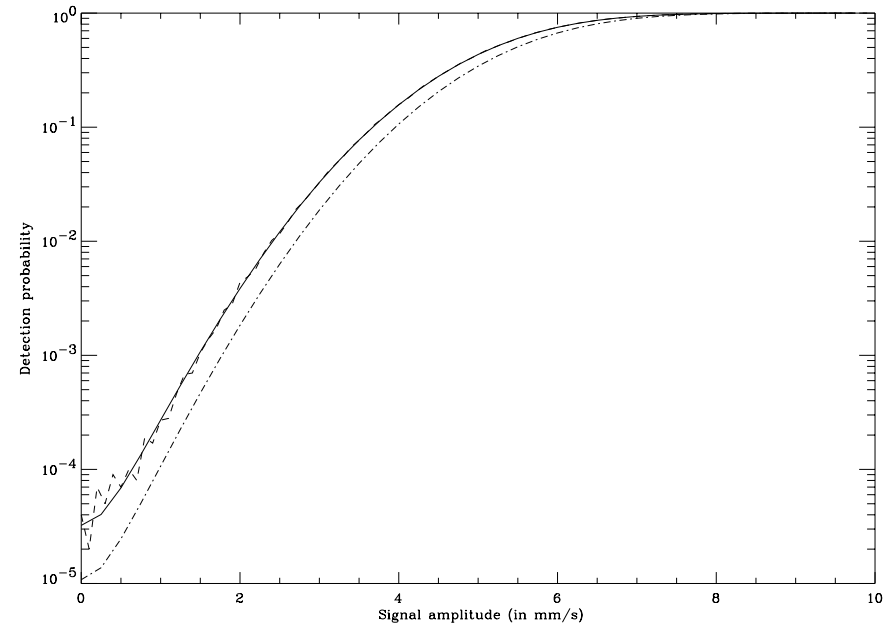

Fig. 5. Detection probability of a sine wave of a given amplitude buried in noise $\left(\sigma=110(\mathrm{~m} / \mathrm{s})^{2} / \mathrm{Hz}\right.$, level of noise around $\left.300 \mu \mathrm{Hz}\right)$, with $z_{\text {det }}$, the $90 \%$ confidence level in a $\mathrm{x} 5$ oversampled spectrum, given by $11.4 \sigma$. The solid line represents the analytic calculation. The dashed line represents the result of Monte-Carlo simulations. The dashed-dot line is the calculation for a 5-times oversampled timeseries.

hypotheses are not in conflict. They are separate contributions to the decision process (Papoulis 1991). The probability that a sine wave of amplitude $A$ is detected above the $10 \%$ limit in a frequency window $\Delta v$ over a time series of length $T$ is given by Eq. (7), with $z_{0}=z_{\text {det }}$, where $z_{\text {det }}$ has to be chosen according to the detection strategy.

Figure 5 shows the detection probability for a $20 \mu \mathrm{Hz}$ band and a 1888-day time series. This is the probability that a signal of a given amplitude $A$ exceeds a given $z_{\mathrm{det}}$. In this example, $z_{\mathrm{det}}$ has been chosen as the $90 \%$ confidence level in an oversampled spectrum. The assumed level of noise is $\sigma=110(\mathrm{~m} / \mathrm{s})^{2} / \mathrm{Hz}$ in the power spectrum. Figure 5 shows that a sine wave with an amplitude of $4 \mathrm{~mm} / \mathrm{s}$ would have a $10 \%$ chance of detection, whilst a signal larger than $8 \mathrm{~mm} / \mathrm{s}$ would invariably be detected.

The implication of this result is that we cannot dismiss the possibility of a potential candidate being real, merely because other $g$ modes, expected to have a comparable intensity, are absent. More specifically, the appearance of only one member of a predicted multiplet is a normal situation and should not be taken to imply that it is a false identification.

\subsection{Single or averaged spectra}

Another approach in computing Fourier spectra is simply to cut the time series into independent sub-series, to compute their separate spectra and to average the results. The aim of this is to decrease the influence of the noise. However, we show here that averaging will not improve the signal to noise ratio, in the case of the present assumption regarding the nature of the signal. Using the normalization implied by Eq. (1), we define here the signal-to-noise ratio $S N R$ as the expected height of the peak in $\sigma$ units at the frequency $v_{0}$ :

$S N R=\frac{P_{S}+P_{\mathcal{N}}}{P_{\mathcal{N}}}=1+N\left(\frac{A_{0}}{2 \sigma_{0}}\right)^{2}$ 


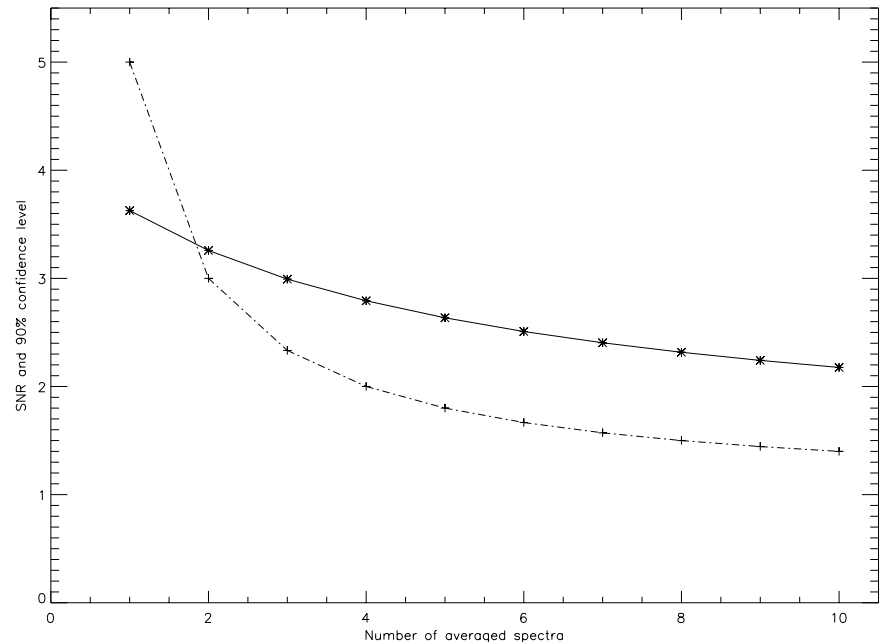

Fig. 6. Ratio of the $90 \%$ significance level to the expected level of noise versus the number of degrees of freedom (two times the number of spectra averaged) (solid curve) compared to the signal to noise ratio (dashed curve).

Now consider the case of the average of $M$ spectra of subsets $k$ of the data, each having $N / M$ points and amplitudes $A_{0}$ :

$P_{S}^{\prime}=\frac{1}{M} \sum_{k=0}^{M-1} \frac{N \delta t}{M}\left(\frac{A_{0}}{2}\right)^{2}$

and $P_{\mathcal{N}}^{\prime}=\frac{1}{M} \sum_{k=0}^{M-1} \sigma_{0}^{2} \delta t$,

giving $S N R^{\prime}=1+\frac{N}{M}\left(\frac{A_{0}}{2 \sigma_{0}}\right)^{2}$

which shows that $S N R$ decreases as the number of sub-series increases. The attraction of averaging is to decrease the variance of the noise power, which makes the signal more "visible". However, this is offset by the variation with $M$ of a given level of confidence ( $90 \%$ for example), here expressed in $\sigma$ units to make a comparison with the SNR easier. Levels of confidence must be recomputed with a statistical law, $\chi_{2 \mathrm{M}}^{2}$, adapted to the averaged spectra, which requires a numerical estimation of confidence levels. Figure 6 compares the variation of the SNR defined above with the $90 \%$ confidence level, each plotted against the number of averaged spectra. It shows that the $S N R$ decreases faster than the $90 \%$ confidence level when increasing the number of averaged spectra. This, added to the loss of frequency resolution implied by the use of shorter time series, leads to our opinion that this strategy is not to be recommended for the purpose of isolating $g$ modes, despite the decrease in the variance of the noise power. However, in defence of the method, we note that some workers find that the modified statistics renders more robust the procedure for fitting Lorentzian or asymmetric profiles (important for typical $p$ modes).

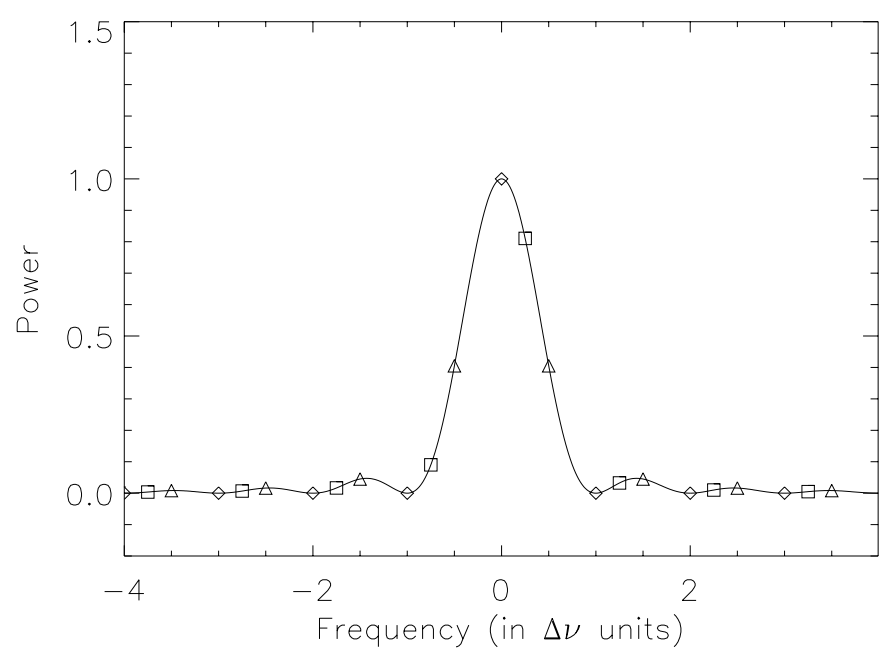

Fig. 7. The solid line represents the continuous spectrum of a sine wave observed during a finite duration, yielding the sinc profile. The squares/diamonds/triangles are different possible sampling of a discrete Fourier transform (the diamonds being the "optimum choice", the triangles the worst one).

\subsection{Frequency sampling}

The discretization in frequency of the FFT has a non negligible effect on the power spectrum of a signal. If the signal we are seeking has no detectable intrinsic width in frequency (as in the case of a continuous sine wave, the expected behaviour of the $g$ modes), its Fourier spectrum is a squared sinc function, caused by the windowing due to the finite duration of the observation, whose maximum occurs at the frequency $v_{0}$ of the sine wave. However, $v_{0}$ might not coincide with a frequency sample $v_{i}$ in the discrete sampling (see Fig. 7). If the real power (in a continuous spectrum) of the signal is $P_{0}$, the power in the discrete spectrum varies between $P_{0}$ and $(2 / \pi)^{2} P_{0} \approx 0.4 P_{0}$, corresponding to the worst case where $v_{i}-v_{0}=\Delta v / 2$ (where $\Delta v$ is the interval between two samples of the spectrum $)^{1}$.

It is then tempting to estimate $P_{0}$ by over-sampling the spectrum (by, for example performing an FFT on an the timeseries elongated by added zeros). However, in this case, the simple statistics described above can no longer be applied. An alternative technique is to make the resonance coincide exactly with a sampling bin, by progressively shortening the timeseries. Here again it is necessary to re-examine the statistics used for the noise, since we are selecting between multiple realizations of the signal plus noise.

Initially, we planned to work on the normal FFT in order to have a clear understanding the statistics. However, although our knowledge of the noise background would be good, it is regrettable that any real sinusoidal signals present would be subjected to an uncertain reduction in power of from $0 \%$ to $60 \%$. For this reason we have chosen instead to over-sample the time series and, by this method, to limit the uncertain reduction in power imposed on any real resonances. This is achieved by increasing the length of the series by a factor $n$ (which we call the padding factor, by adding zeros onto the end of the time-series).

${ }^{1}$ Of course, if noise is present, this attenuation factor is modified. 
Table 1. Showing how the limiting value of the unknown decrease in power of a sine-wave signal will depend on the degree of oversampling (padding) of the initial time-series.

\begin{tabular}{ccc}
\hline \hline padding factor & $\begin{array}{c}\text { possible power decrease } \\
(\%)\end{array}$ & factor $p$ \\
\hline & 60 & 1.0 \\
1 & 9 & 2.4 \\
3 & 3.3 & 2.8 \\
5 & 2.3 & 2.9 \\
6 & 1.7 & 2.9 \\
7 & & \\
\hline
\end{tabular}

Table 1 shows how the power uncertainty due to this sampling effect depends on the over-sampling factor $n$.

The choice of a padding factor of $\times 5$, giving a power uncertainty of $3 \%$, is adopted in this work as a reasonable compromise between the reduced power uncertainty and the increased computing time.

Having chosen a factor of $\times 5$ data-padding, with the consequent factor of 5 redundancy in the resulting FFT, it is now necessary to re-evaluate the statistics to be applied to the noise power redistribution. An analytical solution to this question not being available, a series of Monte Carlo simulations was carried out using Gaussian noise. It was found that, to a high degree of reliability, the modified statistics to take account of a padding factor of $n$ can be obtained by simply multiplying the number of original (unpadded) bins $N$ in the frequency interval considered, by a constant factor $p$, which is a function of $n$. Equation (6) for the probability of at least one peak having a power greater than $m$ then becomes

$P_{N}(m)=1-\left(1-\mathrm{e}^{-m}\right)^{p N}$.

The factor $p$, also listed in Table 1, was found to increase with the padding factor $n$ towards a limiting asymptotic value of 3.0.

The effect of padding on the statistics can be seen to be significant, but not large, owing to the exponential in Eq. (12). For the parameters used in the present analysis (a time-series length of over 5 years and a $20 \mu \mathrm{Hz}$ test bandwidth) the threshold for $10 \%$ probability is increased due to the $\times 5$ padding from $10.3 \sigma$ to $11.4 \sigma$.

\subsection{Length of the series}

As with many helioseismic observations, GOLF is continuing to function, and the time series is continuing to grow in length. Questions can be posed concerning the evolution with time of the credibility of certain candidates. If the time series increases by a certain factor, do we expect the signal-to-noise ratio of the more prominent candidates to increase or decrease? How does this differ according to whether they are real resonances or only chance peaks in the noise background? A number of simulations have been carried out in order to provide statistical answers. The range of questions posed can be quite wide. Here we consider only two cases, which may be of use later in discussing how to interpret our observations.

As is to be expected for the simulated Gaussian background, a certain number of peaks are to be found above the limit of $10 \%$ probability to find at least one peak within a band of $20 \mu \mathrm{Hz}$. In addition, we have introduced some simulated pure sine-wave resonances, with a signal to noise power level ratio of 7, the peaks of many of which appear above this value. If we now double the duration of the series, what happens to these initially detected peaks of both types? The simulations have been carried out using spectra with $\times 5$ padding. The initial conditions correspond with the parameters of the GOLF situation for a half of the present duration of the data-set used. We identify all frequency peaks larger than a threshold, equivalent to a $10 \%$ probability of being due to noise only, within a test frequency band of $20 \mu \mathrm{Hz}$. Some of these arise from the noise (false candidates) and some from the added sine-wave signals (true candidates). 700 independent series have been examined, involving observations of more than 600 peaks. We then examine the spectrum again after the time-series length has doubled to arrive at the present GOLF length. The parameter examined is the probability that the signal is not noise (i.e. 1 minus the probability that it is noise). Of the false candidates, $8 \%$ increase in probability, whilst $92 \%$ decrease (this includes $91 \%$ which fall below the detection threshold). Of the true candidates, $78 \%$ increase in probability, whilst $22 \%$ decrease (including $12 \%$ which fall below the threshold). Tests carried out using different values of initial threshold give broadly similar figures.

We can conclude from these simulations, that a candidate that increases in probability after doubling the length of the series is unlikely to be a false identification coming from the Gaussian background noise.

\subsection{Combining instruments}

It is sometimes claimed that the final verification of a suspected candidate mode will come from combining series from different instruments, especially those on SOHO. This would certainly improve the signal-to-noise ratio, if the dominant noise were due to photons or instrument noise. However, since the largest noise component comes from the Sun itself, and all instruments are regarding the same Sun during the same epoch, such a verification is not obvious. Nevertheless, some useful corroboration might be obtained, if it can be established that different instruments observe the Sun in a basically different manner.

\section{Results}

Following the arguments developed in the previous sections, our approach is to analyse the FFT obtained from the timeseries of more than 5 years, with a $\times 5$ zero padding, and filling also the more important gaps with zeros. The spectra obtained are then analysed for their statistical significance in separate frequency bands of $20 \mu \mathrm{Hz}$ throughout the low-frequency region of interest.

The result is shown in the Fig. 8 for the 12 sections of the frequency spectrum. The ordinates are in units of the local $\sigma$ for each frequency band. The horizontal broken lines represent the probability limits for one bin to exceed this level in that particular frequency band, using the statistics presented in Sects. 4 and 5.3. If all of the data were random Gaussian noise, 

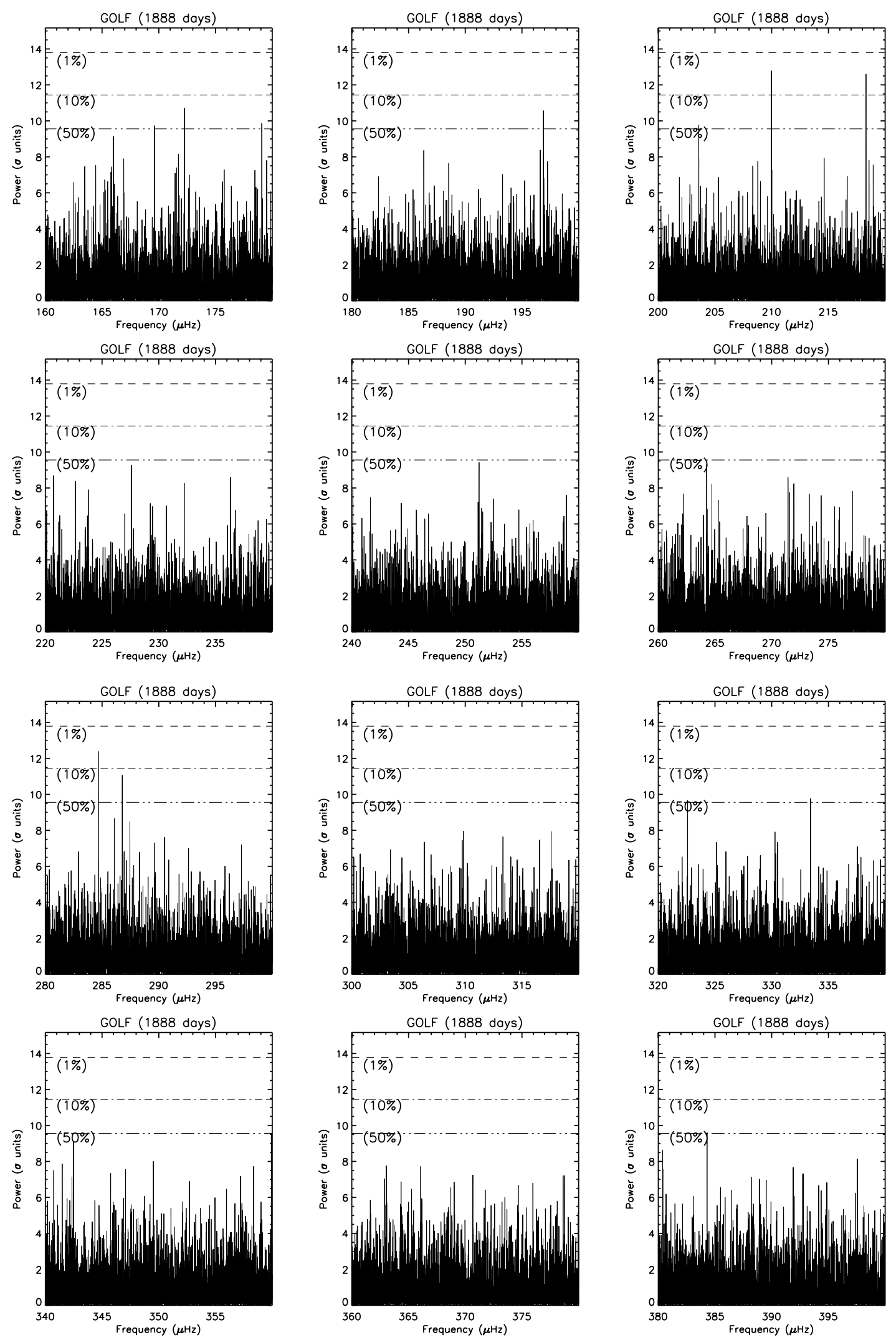

Fig. 8. Showing the spectrum with the limits of probability that the peaks arise from noise alone. 
Table 2. Observed peaks showing a probability to be signal of $>70 \%$ in a $20 \mu \mathrm{Hz}$ test band.

\begin{tabular}{lcc}
\hline \hline $\begin{array}{l}\text { frequency } \\
(\mu \mathrm{Hz})\end{array}$ & $\begin{array}{c}\text { power } \\
\text { (units of } \sigma \text { ) }\end{array}$ & $\begin{array}{c}\text { probability of signal } \\
(\%)\end{array}$ \\
\hline 196.891 & 10.6 & 78 \\
209.974 & 12.8 & 97 \\
218.314 & 12.6 & 97 \\
284.666 & 12.4 & 96 \\
286.781 & 11.0 & 86 \\
\hline
\end{tabular}

we would expect between 4 and 8 of the twelve bands to show at least one peak above the $50 \%$ level. In fact this occurs in 5 of the bands. We would expect between 0 and 2 of the bands to show at least one peak above the $10 \%$ level. This occurs in 2 bands. Statistically, none of the bands should have a peak above the $1 \%$ level; this is the case. Thus far, we could conclude that our probability figures are verified as reasonable, on the hypothesis that all of what we observe is noise.

In Table 2 are listed all of the peaks coming above the $70 \%$ signal probability level. There are 5. Statistically, we would expect between 2 and 6 .

At this point we recall that our calibration methods lead to an uncertainty of the order $12 \%$ in the power level of the higher peaks. This translates into an uncertainty in the percentage probability figures quoted in Table 2 . The translation is very non-linear, as can be seen from the probability limits plotted in Fig. 8. It implies an uncertain precision for peaks that fall above the $90 \%$ probability limit (indicated as the $10 \%$ limit in Fig. 8).

\section{Discussion}

In the foregoing, we have presented a number of arguments for believing that the analysis of a $\times 5$ zero-padded FFT of the total time-series available is a reliable and well-understood approach. In this paper we have chosen to make the minimum of assumptions regarding the Sun or the nature of the $g$ modes expected, and simply to analyse the observations for the probability of identifying statistically significant peaks, occupying one bin in the power spectrum. We are obliged to conclude that there is no strong evidence that any of the peaks reported in Table 2 are other than peaks due to the background noise spectrum. However, the statistical analysis presented in the foregoing sections shows that there is also a realistic possibility that some might indeed be due to solar resonances.

In an earlier analysis (Gabriel et al. 1998), two multiplets near 220 and $252 \mu \mathrm{Hz}$, were reported as possible "candidates" for $g$ modes. This identification did not adopt the same objective criteria used in the present paper. It was based on the search for multiplets in a time/frequency representation. To maintain a consistent basis, we have here re-analysed the first 689 days of data, based on the present approach and give in Table 3 the frequencies and probabilities obtained for the stronger peaks observed. These two earlier candidates had probabilities well below $50 \%$ at that time. Comparison of the two sets shows that
Table 3. Observed peaks using only the first 689 days of data. The precision is related to a bin separation of $17 \mathrm{nHz}$.

\begin{tabular}{lcc}
\hline \hline $\begin{array}{l}\text { frequency } \\
(\mu \mathrm{Hz})\end{array}$ & $\begin{array}{c}\text { power } \\
\text { units of } \sigma)\end{array}$ & $\begin{array}{c}\text { probability of signal } \\
(\%)\end{array}$ \\
\hline 220.71 & 7.8 & $<50$ \\
251.22 & 4.4 & $<50$ \\
252.50 & 5.6 & $<50$ \\
284.66 & 10.6 & 91.7 \\
322.59 & 9.06 & 65.9 \\
\hline
\end{tabular}

they have increased somewhat in significance, but are still below the $50 \%$ level.

Only one peak remains at high significance both in the 1998 analysis and at present, having increased from $92 \%$ to $96 \%$ over that time. This $284.66 \mu \mathrm{Hz}$ resonance has already been identified provisionally as one component of the $n=1, l=1$ multiplet (García et al. 2001b). Even if this identification were to be accepted, the difficulty is the inability to say which it is of the two expected components of the multiplet. According to Provost et al. (2000), the rotational splitting for this particular $p$ mode, which is a deeply penetrating mode with some $g$-mode characteristics, is expected near $0.4 \mu \mathrm{Hz}$. Since the $m=0$ component will not be seen in the GOLF global view, this leaves the $m=1$ and $m=-1$ components with an expected separation of the order $0.8 \mu \mathrm{Hz}$. No resonances of a significant level can be found within $\pm 1.0 \mu \mathrm{Hz}$ of $284.66 \mu \mathrm{Hz}$. This situation is not in disagreement with statistical expectations, however, whether or not the identification is correct.

Leaving aside the $284.66 \mu \mathrm{Hz}$ resonance as a possible $p$ mode, there is no significant identification of a $g$-mode resonance. It is useful now to try to set an upper limit on the amplitude of any $g$ mode present. However, the value we obtain is bound to depend on which of the two hypotheses defined in Sect. 5.1 we adopt to pose the question. With the H0 hypothesis that the time-series is made entirely of noise, the probability that a noise peak reaches a given probability level (here $10 \%$ in the power spectrum, in a $20 \mu \mathrm{Hz}$ interval around $300 \mu \mathrm{Hz}$ ) is around $5.6 \mathrm{~mm} / \mathrm{s}$. This approach has been adopted by other workers in the field (e.g. Appourchaux et al. 2000). The alternative $\mathrm{H} 1$ test is to ask what is the probability that an imbedded coherent sine wave reaches this confidence level, as a function of its amplitude. It yields the result that a signal with an amplitude of $7 \mathrm{~mm} / \mathrm{s}$ would have a $90 \%$ chance to reach the aforementioned " $90 \%$ confidence" level set by the H0 test. This is illustrated in Fig. 5.

It is appropriate to question a basic assumption made in the present analysis. This is that the low-frequency $g$ modes sought will have an intrinsic width of less than one resolution bin in the spectrum. In spite of a very long damping time, slow changes in the resonant cavity, due for example to solar cycle effects, could result in frequency shifts, leading to significant apparent line widths. Such effects, if present, would seriously complicate our analysis, and invalidate the present underlying statistical assumptions. 


\section{Conclusion}

A critical statistical analysis of over five years of GOLF data has shown no significant evidence of the presence of $g$ modes. An upper limit for their velocity amplitudes at the solar surface is found to be around $6 \mathrm{~mm} / \mathrm{s}$, subject to the basic assumptions on the nature of the modes, on which the present study is based. This is consistent with the conclusions of Appourchaux et al. (2000) who set an upper limit to $g$-modes amplitudes at $10 \mathrm{~mm} / \mathrm{s}$.

From Table 2, three possibly significant peaks are found in the frequency range studied: 209.974, 218.374, and $284.666 \mu \mathrm{Hz}$, of which only the last was significant in the first 2 years of data. This is tentatively identified as one member of the $m= \pm 1$ multiplet due to the $n=1, l=1 p$ mode at $284.666 \pm 0.006 \mu \mathrm{Hz}$, in accordance with our previous report (García et al. 2001b). This low-order $p$ mode has a strong $g$-mode quality with an important contribution from the core (Provost et al. 2000) and would therefore be a critical indicator of the solar core conditions. Unfortunately, even if we accept the identification, our inability to confirm which component we see, and the actual splitting, is limiting its usefulness.

Some components from the two multiplets, tentatively identified as $g$-mode candidates by the GOLF team after 2 years of data, are again observed with the present 5 years of data. Although their statistical significance has increased slightly, they remain at a level which does not justify their identification as $g$ modes.

The present analysis has deliberately avoided assumptions based on the physics of the solar interior. The search for $g$ modes might be helped by the inclusion of some of this physics. A complementary study is in hand (Turck-Chièze et al., submitted to ApJ), which aims to exploit the statistical advantages of searching for multiplet structures.

Finally, there remains the possibility to improve the statistics by combining data from different instruments, including those on board of SOHO. This may not be simple to apply, since the major noise component (solar noise) may be common to many instruments. It is nevertheless an avenue of research which should be further explored.

Acknowledgements. The GOLF instrument has been built by a consortium of institutes, involving the participation of many engineers and scientists, as enumerated in our earlier instrument publication (Gabriel et al. 1995). The high quality of the GOLF data is due equally to the outstanding performance of the SOHO platform and overall system. The authors express their appreciation for the key role played by the Project Scientist Gerard Grec in all aspects of the GOLF programme, including the development of the instrumentation and the analysis of the data. We are grateful to Ludovic Sanchez for valuable work on the Monte Carlo evaluation of probabilities for the zeropadded series.

SOHO is a project of international collaboration between ESA and NASA.

\section{Appendix A: The $\mathrm{H} 1$ hypothesis}

This Appendix aims to calculate the probability that a sine wave, combined with a background noise spectrum, reaches a given value in the power spectrum. Specifically, it evaluates the probability of detection, as a function of the amplitude of the sine wave $A$ and the assumed detection level $z_{0}$, for a background Gaussian noise level of variance $\sigma_{0}^{2}$, with a sampling time $\delta t$.

In the presence of a sine wave buried in noise, each Fourier component still has a Gaussian distribution. If the definition of Eq. (1) is chosen for the power spectrum, the power distribution can be written as:

$$
\begin{aligned}
\mathcal{P}(x, y)= & \frac{1}{\pi \sigma_{0}^{2} \delta t} \exp \left(-\left(x-\frac{\sqrt{N \delta t} A}{2} \cos \alpha\right)^{2}\right. \\
& \left.-\left(y-\frac{\sqrt{N \delta t} A}{2} \sin \alpha\right)^{2}\right) / \sigma_{0}^{2} \delta t S
\end{aligned}
$$

where $x$ and $y$ are random variables representing respectively the real and the imaginary part of the Fourier spectra, $\sigma_{0}^{2}$ is the variance of the noise in the temporal domain (corresponding to the level of noise in power), $N$ is the number of points of the time series, and $A$ and $\alpha$ are respectively the amplitude of the sine wave and its phase. Then

$P\left(z<z_{0}, A\right)=$

$\frac{1}{\pi \sigma_{0}^{2} \delta t} \iint_{\mathcal{D}} \exp \left(-\left(x^{2}+y^{2}+\frac{N \delta t A^{2}}{4}-\sqrt{N \delta t} A x\right) / \sigma_{0}^{2} \delta t\right) \mathrm{d} x \mathrm{~d} y$.

In polar coordinates, and using the variable $\beta=\sqrt{N} A / \sigma_{0}$, we have:

$P\left(z<z_{0}, \beta\right)=\frac{\mathrm{e}^{-\frac{\beta^{2}}{4}}}{2 \pi} \int_{0}^{\frac{z_{0}}{\sigma_{0}^{2} \delta t}} \int_{0}^{2 \pi} \exp -(u-\beta \sqrt{u} \cos \theta) \mathrm{d} u \mathrm{~d} \theta$.

It follows that the probability that this sine wave results in a spectral power greater than a level $z_{\operatorname{det}}$ is $1-P\left(z<z_{\operatorname{det}}, \beta\right)$.

\section{References}

Abrams, D., \& Kumar, P. 1996, ApJ, 472, 882

Appourchaux, T. 1998, in Proc. SOHO 6/GONG 98 Workshop, ESA SP-418, 37

Appourchaux, T., Fröhlich, C., Andersen, B. N., et al. 2000, ApJ, 538, 401

Basu, S., Turck-Chièze, S., Berthomieu, G., et al. 2000, ApJ, 35, 1078

Bertello, L., Varadi, F., Ulrich, R. K., et al. 2000, ApJ, 537, 143

Bertello, L., Henney, C. J., Ulrich, R. K., et al. 2000, ApJ, 535, 1066

Chaplin, W. J., Elsworth, Y., Howe, R., et al. 1996, Sol. Phys., 168, 1

Delache, P., \& Scherrer, P. 1983, Nature, 306, 651

Duvall, T. L. Jr., Jefferies, S. M., Harvey, J. W., et al. 1993, ApJ, 410, 829

Fröhlich, C., Andersen, B. N., Appourchaux, T., et al. 1997, Sol. Phys., 170,1

Fröhlich, C., Finsterle, W., Andersen, B. N., et al. 1998, ESA SP-418, 67

Gabriel, A. H., Grec, G., Charra, J., et al. 1995, Sol. Phys., 162, 61

Gabriel, A. H., Charra, J., Grec, G., et al. 1997, Sol. Phys., 175, 207

Gabriel, A. H., Turck-Chièze, S., García, R. A., et al. 1998, in Proc. SOHO 6/GONG 98 Workshop, ESA SP-418, 61

Gabriel, M. 1993, A\&A, 274, 935

García, R. A., Ph.D. Thesis, University of La Laguna, Tenerife, Spain, 1996 
García, R. A., Bertello, L., Turck-Chièze, S., et al. 2001b, Helio- and Asteroseismology at the dawn of the Millenium, ESA SP-464, 473

García, R. A., Régulo, C., Turck-Chièze, S., et al. 2001a, Sol. Phys., 200,361

Gavryusev, V., \& Gavryuseva, E. 1988, Advances in Helio- and Astroseismology, Proc. IAU Symp., 123, 363

Gough, D. O. 1985, in Future missions in solar, heliospheric and space plasma physics, ed. E. J. Rolfe, \& B. Battrick, ESA SP-235, Noordwijk, 183

Grec, G., Fossat, E., Gelly, B., et al. 1991, Sol. Phys., 133, 13

Harvey, J. 1985, in Future missions in solar, heliospheric and space plasma physics, ed. E. J. Rolfe, \& B. Battrick, ESA SP-235, Noordwijk, 199

Harvey, J. W., et al. 1996, Science, 272, 1284

Henney, C. J., Ulrich, R. K., Bertello, L., et al. 1999, A\&A, 348, 627

Hill, H., Fröhlich, C., Gabriel, M., \& Kotov, V. A. 1991, in Solar Interior and Atmosphere, ed. A. N. Cox, W. C. Livingston, \& M. S. Mathews (Univ. Arizona Press), 562

Kumar, P., Quataert, E. J., \& Bahcall, J. N. 1996, ApJ, 458, L83

Pallé, P. L., Régulo, C., Roca Cortés, T., et al. 1999, A\&A, 341, 625

Papoulis, A. 1991, Probability, random variables and stochastic processes, 3rd edition (McGraw-Hill)

Provost, J., Berthomieu, G., \& Morel, P. 2000, A\&A, 353, 775

van der Raay, H. B. 1988, in Proc. Symp. Seismology of the Sun and Sun-like Stars, ESA SP-286 339
Régulo, C., Roca Cortés, T., Boumier, P., et al. 1998, in ed. J. Provost, Proc. IAU Symp. 181, poster volume, OCA Nice, 55

Renaud, C., Grec, G., Boumier, P., et al. 1999, A\&A, 345, 1019

Robillot, J. M., Bocchia, R., \& Denis, N. 1993, in Proc. 5th IRIS Workshop and GOLF'93 Meeting, ed. T. Roca Cortés, \& N. García (I.A.C., April 1993)

Scargle, J. D. 1982, ApJ, 263, 835

Scherrer, et al. 1995, Solar Phys., 162, 129

Thiery, S., Boumier, P., Gabriel, A. H., et al. 2000, A\&A, 355, 743

Thiery, S., Boumier, P., Gabriel, A. H., Henney, C. J., et al. 2001, Helio- and Asteroseismology at the dawn of the Millenium, ESA SP-464, 681

Thomson, D. J., MacLennan, C. G., \& Lanzerotti, L. J. 1995, Nature, 376, 139

Tomczyk, S., et al. 1996, Solar Phys., 159, 1

Toutain, T., Appourchaux, T., Fröhlich, C., et al. 1998, ApJ, 506, L150

Turck-Chièze, S., Brun, A. S., Chièze, J. P., et al. 1998, in Proc. SOHO 6/GONG 98 Workshop, ESA SP-418, 549

Turck-Chièze, S., Couvidat, S., Kosovichev, A. G., et al. 2001, ApJ, 555, L69

Ulrich, R. K., García, R. A., Robillot, J.-M., et al. 2000, A\&A, 364, 799 